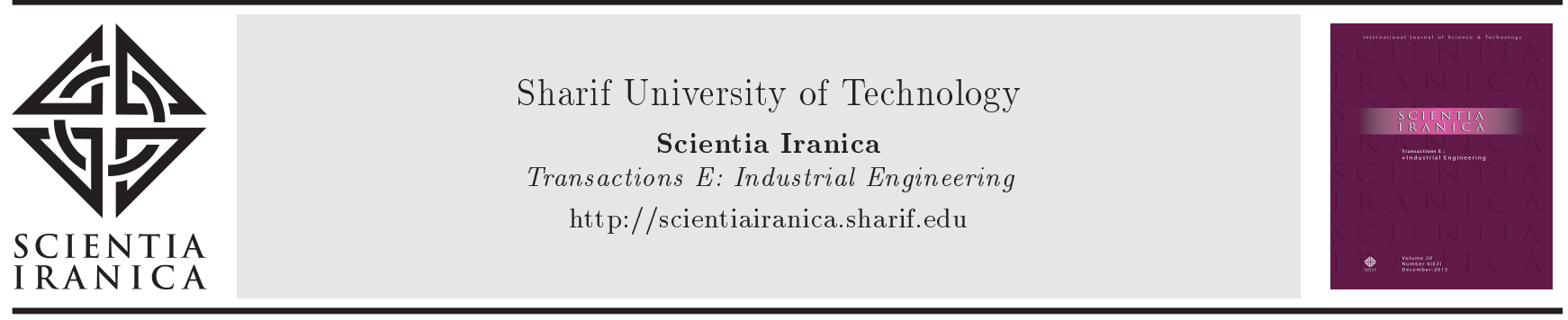

\title{
Identifying the time of step change and drift in phase II monitoring of autocorrelated logistic regression profiles
}

\author{
M.R. Maleki ${ }^{a}$ A. Amiri ${ }^{a, *}$, and A.R. Taheriyoun ${ }^{b}$ \\ a. Department of Industrial Engineering, Faculty of Engineering, Shahed University, Tehran, P.O. Box 18151-159, Iran. \\ b. Department of Statistics, Faculty of Mathematical Sciences, Shahid Beheshti University, G.C., Tehran, P.O. Box 19839-69411, \\ Iran.
}

Received 8 June 2016; received in revised form 21 June 2017; accepted 28 August 2017

\section{KEYWORDS \\ Within-profile autocorrelation; Step change point; \\ Linear trend disturbance; \\ Binary profile; \\ Phase II.}

\begin{abstract}
In some profile monitoring applications, the independency assumption of consecutive binary response values within each profile is violated. To the best of our knowledge, estimating the time of a change in the parameters of an autocorrelated binary profile is neglected in the literature. In this paper, two maximum likelihood estimators are proposed to estimate the real time of step changes and drift in Phase II monitoring of binary profiles in the case of within-profile autocorrelation. Our proposed estimators identify the change point not only in the autocorrelated logistic regression parameters, but also in autocorrelation coefficient. The performance of the proposed estimators to identify the time of change points either in regression parameters or autocorrelation coefficient is evaluated through simulation studies. The results, in terms of the accuracy and precision criteria, show the satisfactory performance of the proposed estimators under both step changes and drift. Moreover, a numerical example is given to illustrate the application of the proposed estimators.
\end{abstract}

(C) 2018 Sharif University of Technology. All rights reserved.

\section{Introduction}

In some manufacturing or nonmanufacturing systems, the relationship between a response variable and one or several explanatory variables, referred to as profile, should be monitored during the time. Concerning the type of this relationship, the profiles are categorized into different classes, such as simple linear, multiple linear, polynomial, nonlinear, Generalized Linear Models (GLM)-based profiles, etc.

Most profile monitoring approaches in the literature have been provided under normality assumption

*. Corresponding author. Tel.: +982151212023

E-mail addresses: reza.maleki@shahed.ac.ir (M.R. Maleki); amiri@shahed.ac.ir (A.Amiri); a_taheriyoun@sbu.ac.ir (A.R. Taheriyoun).

doi: $10.24200 /$ sci.2017.4466 of the response variable. However, in many statistical monitoring applications, the normality assumption of the response variables is violated. In such situations, the generalized linear models are usually used to cover this kind of profiles when the response variable belongs to the family of exponential distributions such as binary, binomial, Poisson, and gamma. In the first research concerning the monitoring of GLM profiles, Yeh et al. [1] extended five Hotelling's $T^{2}$ charts, namely, $T_{H}^{2}, T_{R}^{2}, T_{I}^{2}, T_{E}^{2}$, and $T_{D}^{2}$, for Phase I monitoring of binary profiles. Through simulation studies, they compared the performances of their proposed charts to detect out-of-control disturbances in terms of the signal probability criterion and found that $T_{I}^{2}$ chart outperformed the others. After that, some efforts have been put into monitoring GLM profiles in Phases I and II. Phase I monitoring of GLM profiles is documented as follows.

Amiri et al. [2] evaluated two of the best control 
charts of Yeh et al. [1] including $T_{I}^{2}$ and $T_{R}^{2}$ methods for Phase I monitoring of Poisson regression profiles considering step shifts and drifts. Amiri et al. [3] extended Hotelling's five $T^{2}$ charts for monitoring gamma response profiles in Phase I. They implemented simulation studies and evaluated the performance of their proposed methods under different magnitudes of step shift and drift in terms of the signal probability criterion. Paynabar et al. [4] proposed a general riskadjusted control chart for monitoring binary surgical responses based on the Likelihood Ratio Test (LRT). Noorossana and Izadbakhsh [5] utilized multinomial logistic regression and extended some methods in order to monitor profiles in the case of multinomial response variable. Amiri et al. [6] extended three methods, namely, Hotelling's $T^{2}$, LRT, and $F$ charts, to monitor GLM regression profiles concerning the Poisson response variables in Phase I. A change point detection problem was studied by Shadman et al. [7] to monitor the generalized linear profiles for a large class of response variables with continuous and nominal scales in Phase I. They compared their proposed approach with the existing charts in especial case of binomial and Poisson profiles. Phase II monitoring of GLM profiles in the literature is also addressed as follows.

Izadbakhsh et al. [8] proposed three methods based on the Ordinal Logistic Regression (OLR) to monitor the profiles with ordinal response variable in Phase II. They assessed the performance of the proposed control charts in terms of Average Run Length (ARL) criterion. Shang et al. [9] proposed a scheme to monitor the logistic regression profiles in the case of random explanatory variables. Their proposed method could also detect the mean shifts in the explanatory variables along with the regression parameters. Saghaei et al. [10] proposed two methods including EWMA2 and Hotelling's $T^{2}$ control charts for Phase II monitoring of logistic profiles under binary responses. Four methods, namely, Multivariate Exponentially Weighted Moving Average (MEWMA), $\chi^{2}$, Exponentially Weighted Moving Average (EWMA) with $R$ statistic, and a combination of the last two statistics, were proposed by Noorossana et al. [11] to monitor OLR profiles in Phase II. Soleymanian et al. [12] proposed four control charts including Hotelling's $T^{2}$, MEWMA, LRT, and LRT/EWMA to monitor profiles with binary response variable in Phase II. Noorossana et al. [13] studied monitoring of profiles with multinomial response variables based on multinomial logistic regression. They converted the multinomial logistic regression to the Poisson GLM using $\log$ link and proposed two methods including MEWMA and LRT statistics to monitor multinomial logistic regression profiles in Phase II. Using three approaches including LRT, MEWMA, and Support Vector Machine (SVM), the Phase II monitoring of logistic regression profiles was studied by Noorossana et al. [14]. Shadman et al. [15] introduced a unified framework based on Rao score test to monitor GLM profiles in Phase II. Some different control charts to monitor the shape parameter of a Weibull regression model have been studied by Panza and Vargas [16] in Phase II. Imani and Amiri [17] extended two methods, namely, $T^{2}$ based chart and Skinner's residual based chart, to monitor the logistic regression profiles in a two-stage process. A unified framework was proposed by Qi et al. [18] based on the weighted likelihood ratio test for Phase II monitoring of generalized linear profiles either for the fixed explanatory variables or for the random ones.

Once a monitoring statistic falls outside the control limit intervals, a signal will be received from the control chart. However, identifying the real time when the process first goes to an out-of-control situation helps the quality practitioners to eliminate the sources of the signals more effectively. Recently, several researches have been done to estimate the real time of change, referred to as the "change point," in linear profiles. However, the literature on estimating the change point of GLM profiles is not large enough. Sharafi et al. [19] presented the Maximum Likelihood Estimator (MLE) to estimate the real time of a step change in Phase II monitoring of binary profiles. Afterwards, Sharafi et al. [20] used MLE approach to estimate the change point in binary profiles when the type of change was drift. The ML estimator was developed by Sharafi et al. [21] to identify the change point in Phase II monitoring of Poisson regression profiles. Zand et al. [22] extended two methods, namely, LRT and clustering, to estimate the real time of a step change in Phase I monitoring of binary profiles. Sogandi and Amiri [23] estimated the real time of a step change in Phase II monitoring of gamma regression profile using MLE approach. The change point estimation of gamma regression profiles with a linear trend disturbance based on the extension of MLE approach was studied by Sogandi and Amiri [24]. Sogandi and Amiri [25] used a general MLE approach to estimate the change point of GLM profiles under the monotonic change in Phase II. Shang et al. [26] explored the change-point estimation of binary profiles in Phase II based on LRT method by considering random explanatory variables.

In all of the above-mentioned researches, either on monitoring or on change point estimation of GLM profiles, the response variables within each profile have been assumed independent. This assumption is satisfied under very restricted laboratory conditions and there is no guarantee that it holds in practice. For instance, consider the manufacturing process of electrolytic capacitors in which the final quality of the units is expressed as "pass" or "fail" and the successive observations are taken in short intervals. In this process, the 
explanatory variables are the type of raw material, level of voltage, frequency, and temperature. Obviously, the binary response observations are autocorrelated due to the short time of measuring the sampled observations within each profile. The binary nature of the response variable induces the use of logistic regression. However, this model strongly depends on the independency assumption of experimental units in different levels of explanatory variables. Consequently, since both normality and independency assumptions are simultaneously violated, the common approaches available in the literature cannot be used for monitoring such autocorrelated GLM profiles. Although it is very likely to face situations with the lack of normality and independency, simultaneously, very little attention has been devoted to monitor autocorrelated GLM profiles. We can mention Koosha and Amiri [27], who investigated the effect of within-profile autocorrelation on monitoring logistic regression profiles in Phase I. They suggested two remedial approaches to account for the effect of autocorrelation in terms of signal probability criterion. Maleki et al. [28] studied Phase II monitoring of autocorrelated binary profiles using a Markov-based model. For this purpose, they introduced a logistic regression model, which took into account the withinprofile autocorrelation. They evaluated the performance of their proposed method in detecting different step shifts in the vector of regression model parameters in terms of ARL criterion. It is worth mentioning that, to monitor autocorrelated binary profiles, it is important to identify the time when the process starts to change to an out-of-control situation after receiving an out-of-control signal. However, to the best of the authors' knowledge, estimating the real time of change in autocorrelated GLM profiles has obviously been neglected in the literature. The mentioned research gap as well as the wide application of the autocorrelated GLM profiles in industrial or service environments is a very good motivation to analyze such profiles. Hence, in this paper, we propose two MLE methods to estimate the real time of change point under step changes and drift, respectively, in Phase II monitoring of autocorrelated logistic regression profiles. We assume that the response values within each profile are autocorrelated and follow first order autoregressive $(\mathrm{AR}(1))$ model. Note that in Maleki et al. [28], only step shifts are taken into account. However, in this paper, not only the step changes but also the linear trends in the vector of model parameter are studied.

The rest of this paper is organized as follows: In the following section, the preliminaries and the model assumptions are described. Two estimators to identify the time of changes in binary profiles considering the within-profile autocorrelation in Phase II are presented in Section 3. The first method estimates the time of change under step changes while the second estimator determines the change point under linear trend in model parameters. Section 4 contains simulation studies to evaluate the performance of the proposed estimators in terms of accuracy and precision criteria. An illustrative example is provided in Section 5 to show the application of our proposed estimators. The concluding remarks and a recommendation for future study are discussed in Section 6 .

\section{Preliminaries and assumptions}

As noted previously, Maleki et al. [28] employed a unified methodology for Phase II monitoring of autocorrelated binary profiles concerning the within-profile autocorrelation. Assume that $y_{i}$ denotes the binary response value at $i$ th; $i=1, \ldots, n$ treatment of a sampled profile. If the response values are autocorrelated within each profile, then $y_{i}$ is affected by both $\mathbf{x}_{\mathbf{i}}=\left(x_{i 1}, x_{i 2}, \ldots, x_{i p}\right)$, the $p$-dimensional explanatory variables, and the observed responses values in the previous experimental settings, i.e., $\left(y_{i-1}, y_{i-2}, \ldots, y_{1}\right)$. Using the logistic regression model to express the relationship between binary response variable and explanatory variable(s) leads to misleading results, because this model ignores the within-profile autocorrelation structure. Considering the first order autoregressive model, i.e., AR(1), between consecutive response values in each profile, Maleki et al. [28] proposed a stationary state space $\{0,1\}$ model for $i$ th treatment as follows:

$$
\begin{aligned}
p_{y_{i-1}}= & P\left(y_{i}=1 \mid y_{i-1}=j\right) \\
= & \left\{\begin{array}{c}
P\left(y_{i}=1 \mid y_{i-1}=0\right)=P_{0} \\
=\frac{1}{1+e^{\mathbf{X}_{i} \boldsymbol{\beta}}} \\
\left(e^{\mathbf{X}_{i} \boldsymbol{\beta}}-\rho e^{\frac{1}{2}\left(\mathbf{X}_{i}+\mathbf{X}_{i-1}\right) \boldsymbol{\beta}}\right) \text { if } y_{i-1}=0 \\
P\left(y_{i}=1 \mid y_{i-1}=1\right)=P_{1} \\
=\frac{1}{1+e^{\mathbf{X}_{i} \boldsymbol{\beta}}} \\
\left(e^{\mathbf{X}_{i} \boldsymbol{\beta}}+\rho e^{\frac{1}{2}\left(\mathbf{X}_{\left.i-\mathbf{X}_{i-1}\right) \boldsymbol{\beta}}\right)}\right) \text { if } y_{i-1}=1
\end{array}\right.
\end{aligned}
$$

where $\rho=\operatorname{Corr}\left(y_{i}, y_{i-1}\right)$, and $\boldsymbol{\beta}=\left(\beta_{1}, \beta_{2}, \ldots, \beta_{p}\right)^{\prime}$ is the vector of regression coefficients. In the second step, they presented the likelihood function by taking into account the within-profile autocorrelation. Maleki et al. [28] noted that the common approaches in the area of monitoring GLM profiles were proposed under the independency assumption of consecutive response values within each profile. Hence, they developed an evolutionary algorithm to estimate the regression parameters by maximizing the likelihood function. Afterwards, they computed the Fisher information matrix of the induced likelihood function under Eq. (1) to estimate the asymptotic covariance matrix of the estimated parameters, $\boldsymbol{\Sigma}_{\hat{\boldsymbol{\theta}}}$. Then, they proposed two 
control charts, namely, the extended Hotelling's $T^{2}$ and MEWMA, to monitor the model parameters under different step shifts.

\section{Proposed change point estimators}

In this section, the proposed estimators to identify the real time of a change in a binary profile when the observations within each profile are autocorrelated and follow AR(1) model are suggested. In Subsection 3.1, the type of disturbance is assumed to be a step change while in Subsection 3.2, the change type is considered as a drift. Note that, first, the Hotelling's $T^{2}$ chart proposed by Maleki et al. [28] for Phase II monitoring of autocorrelated binary profiles is used to detect any faults in the vector of regression parameters. It is worth mentioning that, for $j$ th profile, the $T_{j}^{2}$ statistic considering the within-profile autocorrelation structure is defined as:

$$
T_{j}^{2}=\left(\hat{\boldsymbol{\theta}}_{j}-E(\hat{\boldsymbol{\theta}})\right)^{\prime} \boldsymbol{\Sigma}_{\hat{\boldsymbol{\theta}}}^{-1}\left(\hat{\boldsymbol{\theta}}_{j}-E(\hat{\boldsymbol{\theta}})\right) ; \quad j=1, \ldots, T,
$$

where $\hat{\boldsymbol{\theta}}_{j}=\left(\hat{\rho}_{j}, \hat{\beta}_{1, j}, \hat{\beta}_{2, j}, \ldots, \hat{\beta}_{p, j}\right)^{\prime}$ is a vector of estimated parameters for $j$ th profile and $E(\hat{\boldsymbol{\theta}})=$ $\left(\rho, \beta_{1}, \beta_{2}, \ldots, \beta_{p}\right)^{\prime}$ denotes the vector of expected values for the estimated parameters in the extended logistic regression model. Also, $\boldsymbol{\Sigma}_{\hat{\boldsymbol{\theta}}}$ denotes the covariance matrix of the estimated model parameters. For detailed information, refer to Maleki et al. [28].

\subsection{Proposed estimator under step change}

It is assumed that the model parameters of the autocorrelated binary profile are statistically in-control at the beginning of the underlying process with the known vector of $\boldsymbol{\theta}_{0}=\left(\rho, \beta_{1}, \ldots, \beta_{p}\right)^{\prime}$. After an unknown step change point at $\tau_{s c}$ th profile, the vector of model parameters goes to an out-of-control state denoted by $\boldsymbol{\theta}_{1}$, where $\boldsymbol{\theta}_{1} \neq \boldsymbol{\theta}_{0}$. The underlying process continues until period $T$ when the Hotelling's $T^{2}$ control chart triggers an out-of-control signal. In other words, we have $T_{j}^{2} \leq U C L$ for $j<T$, and $T_{j}^{2}>U C L$ for $j=T$, where $U C L$ denotes the upper control limit of Hotelling's $T^{2}$ chart. Note that the value of $U C L$ is set such that a desired in-control average run length $\left(A R L_{0}\right)$ value is achieved. The ML estimates, $\hat{\boldsymbol{\theta}}$, are asymptotically distributed with multivariate normal distribution with the mean vector of $\boldsymbol{\theta}_{0}$ and covariance matrix of $\boldsymbol{\Sigma}_{\hat{\boldsymbol{\theta}}}$, which is derived based on the inverse of Fisher information matrix. During the formulation of subgroups, when $j=1,2, \ldots, \tau_{s c}$, the vector of model parameters $\boldsymbol{\theta}_{j}$ is equal to its known in-control value $\boldsymbol{\theta}_{0}$. For these subgroups, we have:

$$
\hat{\boldsymbol{\theta}}_{1}, \ldots, \hat{\boldsymbol{\theta}}_{\tau_{s c}} \stackrel{i i d}{\sim} M V N\left(\boldsymbol{\theta}_{0}, \boldsymbol{\Sigma}_{\hat{\boldsymbol{\theta}}}\right) .
$$

As noted, for $j=\tau_{s c}+1, \ldots, T$, the vector of regression parameters is equal to some unknown vector of $\boldsymbol{\theta}_{1}$, where $\boldsymbol{\theta}_{1} \neq \boldsymbol{\theta}_{0}$. In this case, the observations for the out-of-control process follow multivariate normal distribution as:

$$
\hat{\boldsymbol{\theta}}_{\tau_{s c}+1}, \ldots, \hat{\boldsymbol{\theta}}_{T} \stackrel{i i d}{\sim} M V N\left(\boldsymbol{\theta}_{1}, \boldsymbol{\Sigma}_{\hat{\boldsymbol{\theta}}}\right) .
$$

In Eqs. (3) and (4), $\boldsymbol{\theta}_{0}, \boldsymbol{\theta}_{1} \in R^{p+1}, \boldsymbol{\Sigma}_{\hat{\boldsymbol{\theta}}}$ belongs to the family of all symmetric positive definite $(p+1) \times$ $(p+1)$ matrices, and $\tau_{s c} \in\{1,2, \ldots, T-1\}$. We assume that the model parameters remain at the outof-control level until the step change is detected and the corresponding assignable cause is eliminated. Two unknown parameters in the regression model are $\boldsymbol{\theta}_{1}$ and $\tau_{s c}$. Based on the aforementioned explanations, the likelihood function by considering the withinautocorrelation structure is:

$$
\begin{aligned}
L\left(\boldsymbol{\theta}_{1}, \tau_{s c} \mid \hat{\boldsymbol{\theta}}, \boldsymbol{\Sigma}_{\hat{\boldsymbol{\theta}}}\right) & \\
= & \left\{\prod_{j=1}^{\tau_{s c}}(2 \pi)^{-(p+1) / 2}\left|\boldsymbol{\Sigma}_{\hat{\boldsymbol{\theta}}}\right|^{-1 / 2}\right. \\
& \left.\exp \left[-\frac{1}{2}\left(\hat{\boldsymbol{\theta}}_{j}-\boldsymbol{\theta}_{0}\right)^{\prime} \boldsymbol{\Sigma}_{\hat{\boldsymbol{\theta}}}^{-1}\left(\hat{\boldsymbol{\theta}}_{j}-\boldsymbol{\theta}_{0}\right)\right]\right\} \\
& \times\left\{\prod_{j=\tau_{s c}+1}^{T}(2 \pi)^{-(p+1) / 2}\left|\boldsymbol{\Sigma}_{\hat{\boldsymbol{\theta}}}\right|^{-1 / 2}\right. \\
& \left.\exp \left[-\frac{1}{2}\left(\hat{\boldsymbol{\theta}}_{j}-\boldsymbol{\theta}_{1}\right)^{\prime} \boldsymbol{\Sigma}_{\hat{\boldsymbol{\theta}}}^{-1}\left(\hat{\boldsymbol{\theta}}_{j}-\boldsymbol{\theta}_{1}\right)\right]\right\} .
\end{aligned}
$$

The MLE of $\tau_{s c}$ under step changes (denoted by $\hat{\tau}_{s c}$ ) considering the within-autocorrelation structure is the value that maximizes Eq. (5). Simplifying this equation leads to the following result:

$$
\begin{aligned}
L\left(\boldsymbol{\theta}_{1}, \tau_{s c} \mid \hat{\boldsymbol{\theta}}, \boldsymbol{\Sigma}_{\hat{\boldsymbol{\theta}}}\right)=\left((2 \pi)^{-T(p+1) / 2}\right) \times \boldsymbol{\Sigma}_{\hat{\boldsymbol{\theta}}}^{-T / 2} \\
\times\left\{\exp \left[-\frac{1}{2} \sum_{j=1}^{\tau_{s c}}\left(\hat{\boldsymbol{\theta}}_{j}-\boldsymbol{\theta}_{0}\right)^{\prime} \boldsymbol{\Sigma}_{\hat{\boldsymbol{\theta}}}^{-1}\left(\hat{\boldsymbol{\theta}}_{j}-\boldsymbol{\theta}_{0}\right)\right]\right. \\
\left.\quad \exp \left[-\frac{1}{2} \sum_{j=\tau_{s c}+1}^{T}\left(\hat{\boldsymbol{\theta}}_{j}-\boldsymbol{\theta}_{1}\right)^{\prime} \boldsymbol{\Sigma}_{\hat{\boldsymbol{\theta}}}^{-1}\left(\hat{\boldsymbol{\theta}}_{j}-\boldsymbol{\theta}_{1}\right)\right]\right\} .
\end{aligned}
$$

We are supposed to maximize Eq. (6) with respect to $\boldsymbol{\theta}_{1}$ and $\tau_{s c}$ within the mentioned parameter space. To this end, note that since the parameter space concerning $\tau_{s c}$ is countable, we have:

$$
\max _{\tau_{s c}}\left(\max _{\boldsymbol{\theta}_{1}} L\left(\boldsymbol{\theta}_{1} \mid \tau_{s c}, \hat{\boldsymbol{\theta}}, \boldsymbol{\Sigma}_{\hat{\boldsymbol{\theta}}}\right)\right) .
$$

In other words, maximizing Eq. (6) is equivalent to maximizing Eq. (7) for all possible change point values. 
Hence:

$$
\begin{aligned}
\hat{\tau}_{s c} & =\underset{0<\tau_{s c} \leq T-1}{\arg } \max \left(\max _{\boldsymbol{\theta}_{1}} L\left(\boldsymbol{\theta}_{1} \mid \tau_{s c}, \hat{\boldsymbol{\theta}}, \boldsymbol{\Sigma}_{\hat{\boldsymbol{\theta}}}\right)\right) \\
& =\underset{0<\tau_{s c} \leq T-1}{\arg } \max \left(L\left(\tilde{\boldsymbol{\theta}}_{1} \mid \tau_{s c}, \hat{\boldsymbol{\theta}}, \boldsymbol{\Sigma}_{\hat{\boldsymbol{\theta}}}\right)\right)
\end{aligned}
$$

where ([29], p. 67):

$$
\tilde{\boldsymbol{\theta}}_{1}, \tau_{s c}=\frac{1}{T-\tau_{s c}} \sum_{j=\tau_{s c}+1}^{T} \hat{\boldsymbol{\theta}}_{j} .
$$

\subsection{Proposed estimator under linear trend}

Similar to Subsection 3.1, at the beginning of the process, the underlying profile is statistically in-control (for subgroups $j=1, \ldots, \tau_{l t}$ ) with known regression parameters of $\boldsymbol{\theta}_{0}=\left(\rho, \beta_{1}, \ldots, \beta_{p}\right)^{\prime}$. After $\tau_{l t}$ th binary profile, the vector of model parameters linearly changes to an out-of-control vector of $\boldsymbol{\theta}_{j}=\boldsymbol{\theta}_{0}+\mathbf{b}\left(j-\tau_{l t}\right) ; j=$ $\tau_{l t}+1, \tau_{l t}+2, \ldots, T$, where $T$ is the index of profile when the $T^{2}$ chart triggers a signal. Note that vector $\mathbf{b}=\left(b_{\rho}, b_{\beta_{1}}, \ldots, b_{\beta_{p}}\right)^{\prime}$ denotes the slope vector of linear disturbance in $\boldsymbol{\theta}_{0}$. Two unknown parameters in the regression model are vector $\mathbf{b}$ and $\tau_{l t}$. The likelihood function under drift disturbance in the parameters of the underlying autocorrelated binary profile is:

$$
\begin{aligned}
& L\left(\mathbf{b}, \tau_{l t} \mid \hat{\boldsymbol{\theta}}, \boldsymbol{\Sigma}_{\hat{\boldsymbol{\theta}}}\right) \\
&= \prod_{j=1}^{\tau_{l t}}(2 \pi)^{-(p+1) / 2}\left|\boldsymbol{\Sigma}_{\hat{\boldsymbol{\theta}}}\right|^{-1 / 2} \\
& \exp \left[-\frac{1}{2}\left(\hat{\boldsymbol{\theta}}_{j}-\boldsymbol{\theta}_{0}\right)^{\prime} \boldsymbol{\Sigma}_{\hat{\boldsymbol{\theta}}}^{-1}\left(\hat{\boldsymbol{\theta}}_{j}-\boldsymbol{\theta}_{0}\right)\right] \\
& \prod_{j=\tau_{l t}+1}^{T}(2 \pi)^{-(p+1) / 2}\left|\boldsymbol{\Sigma}_{\hat{\boldsymbol{\theta}}}\right|^{-1 / 2} \\
& \exp \left[-\frac{1}{2}\left[\hat{\boldsymbol{\theta}}_{j}-\left(\boldsymbol{\theta}_{0}+\mathbf{b}\left(j-\tau_{l t}\right)\right)\right]\right. \\
&\left.\boldsymbol{\Sigma}_{\hat{\boldsymbol{\theta}}}^{-1}\left[\hat{\boldsymbol{\theta}}_{j}-\left(\boldsymbol{\theta}_{0}+\mathbf{b}\left(j-\tau_{l t}\right)\right)\right]\right] .
\end{aligned}
$$

The MLE of $\tau_{l t}$ under linear trend (denoted by $\hat{\tau}_{l t}$ ) in the regression parameters of the underlying binary profile considering the within-profile autocorrelation is obtained by maximizing Eq. (10). This equation can also be simplified as:

$$
\begin{aligned}
L\left(\mathbf{b}, \tau_{l t} \mid \hat{\boldsymbol{\theta}}, \boldsymbol{\Sigma}_{\hat{\boldsymbol{\theta}}}\right)=\left((2 \pi)^{-T(p+1) / 2}\right) \times \boldsymbol{\Sigma}_{\hat{\boldsymbol{\theta}}}^{-T / 2} \\
\times\left\{\exp \left[-\frac{1}{2} \sum_{j=1}^{\tau_{l t}}\left(\hat{\boldsymbol{\theta}}_{j}-\boldsymbol{\theta}_{0}\right)^{\prime} \boldsymbol{\Sigma}_{\hat{\boldsymbol{\theta}}}^{-1}\left(\hat{\boldsymbol{\theta}}_{j}-\boldsymbol{\theta}_{0}\right)\right]\right.
\end{aligned}
$$

$$
\begin{aligned}
& \exp \left[-\frac{1}{2} \sum_{j=\tau_{l t}+1}^{T}\left[\hat{\boldsymbol{\theta}}_{j}-\left(\boldsymbol{\theta}_{0}+\mathbf{b}\left(j-\tau_{l t}\right)\right)\right]^{\prime}\right. \\
& \left.\left.\boldsymbol{\Sigma}_{\hat{\boldsymbol{\theta}}}^{-1}\left[\hat{\boldsymbol{\theta}}_{j}-\left(\boldsymbol{\theta}_{0}+\mathbf{b}\left(j-\tau_{l t}\right)\right)\right]\right]\right\} .
\end{aligned}
$$

To obtain $\hat{\tau}_{l t}$, an expression for vector $\mathbf{b}$ in terms of $\tau_{l t}$ (denoted by $\hat{\boldsymbol{b}}_{\boldsymbol{\tau}}$ ) should be estimated. Since obtaining this expression is not a trivial task, a new approach based on Least Squares (LS) method is proposed to obtain $\hat{\boldsymbol{b}}_{\boldsymbol{\tau}}$. Obviously, for subgroups $j=\tau_{l t}+1, \ldots, T$ :

$$
\begin{gathered}
\hat{\boldsymbol{\theta}}_{j}=\boldsymbol{\theta}_{j}+\boldsymbol{\varepsilon}_{j}=\boldsymbol{\theta}_{0}+\mathbf{b}\left(j-\tau_{l t}\right)+\boldsymbol{\varepsilon}_{j}, \\
j=\tau_{l t}+1, \ldots, T .
\end{gathered}
$$

It can be statistically checked that $\varepsilon_{j}{ }^{\prime} s ; j=\tau_{l t}+$ $1, \ldots, T$ are independent and identically distributed as:

$$
\varepsilon_{j} \sim M V N\left(\mathbf{0}, \boldsymbol{\Sigma}_{\hat{\boldsymbol{\theta}}}\right)
$$

Here, for fixed value of $\tau_{l t}$, a regression at $\hat{\boldsymbol{\theta}}_{j}-\boldsymbol{\theta}_{0}$ on $\left(j-\tau_{l t}\right)$ is considered. The LS estimate of slope parameter is an ML estimate of $\mathbf{b}$ and obtained as follows:

$$
\hat{\mathbf{b}}_{\boldsymbol{\tau}}=\left(\mathbf{V}^{\prime} \mathbf{V}\right)^{-1} \mathbf{V}^{\prime} \mathbf{U}
$$

where $\mathbf{U}=\left(\hat{\boldsymbol{\theta}}_{\tau_{l t}+1}-\boldsymbol{\theta}_{0}, \hat{\boldsymbol{\theta}}_{\tau_{l t}+2}-\boldsymbol{\theta}_{0}, \ldots, \hat{\boldsymbol{\theta}}_{T}-\boldsymbol{\theta}_{0}\right)^{\prime}$ and $\mathbf{V}=\left(1,2, \ldots, T-\tau_{l t}\right)^{\prime}$ are $\left(T-\tau_{l t}\right) \times(p+1)$ and $\left(T-\tau_{l t}\right) \times 1$ matrices. After obtaining the vector of $\hat{\mathbf{b}}_{\boldsymbol{\tau}}$, we substitute it in Eq. (12) and determine the value of likelihood function for all possible values of the parameter $\tau_{l t}$. Hence, the estimated change point concerning the linear trend in the model parameters is obtained as follows:

$$
\hat{\tau}_{l t}=\underset{0<\tau_{l t} \leq T-1}{\arg } \max \left(\max _{\mathbf{b}} L\left(\mathbf{b} \mid \tau_{l t}, \hat{\boldsymbol{\theta}}, \boldsymbol{\Sigma}_{\hat{\boldsymbol{\theta}}}\right)\right),
$$

where vector $\mathbf{b}$ is estimated according to Eq. (14).

\section{Performance evaluation}

In this section, the performance of the proposed estimators in identifying the time of changes in the regression parameters of the autocorrelated binary profiles in Phase II is evaluated through two numerical examples. In Example 1, the shift type is considered as a step change while in Example 2, the process parameters change by a linear trend.

\subsection{Example 1}

To evaluate the performance of the proposed estimator to identify the time of step change in parameters of Eq. (1), we assume that $\rho=0.15$ and $p=2$. The 
Table 1. Characteristics of estimates under step changes from $\beta_{1}$ to $\beta_{1}+k_{1} \sigma_{\hat{\beta}_{1}}$ with different values of $k_{1}$.

\begin{tabular}{ccccccc}
\hline \multirow{2}{*}{ Criterion } & \multicolumn{7}{c}{ Shift } & $\left(\boldsymbol{k}_{\mathbf{1}}\right)$ \\
\cline { 2 - 7 } & $\mathbf{0 . 2 5}$ & $\mathbf{0 . 5}$ & $\mathbf{0 . 7 5}$ & $\mathbf{1}$ & $\mathbf{1 . 5}$ & $\mathbf{2}$ \\
\hline$E(|\hat{\tau}-\tau|)$ & 61.32 & 15.52 & 3.47 & 1.26 & 0.86 & 0.77 \\
$S t d(|\hat{\tau}-\tau|)$ & 54.81 & 19.27 & 4.63 & 1.56 & 0.99 & 0.73 \\
$P(|\hat{\tau}-\tau|=0)$ & $2.1 \%$ & $10.2 \%$ & $21.2 \%$ & $31.0 \%$ & $35.8 \%$ & $36.1 \%$ \\
$P(|\hat{\tau}-\tau| \leq 1)$ & $4.7 \%$ & $21.6 \%$ & $52.9 \%$ & $74.4 \%$ & $87.6 \%$ & $90.4 \%$ \\
$P(|\hat{\tau}-\tau| \leq 2)$ & $7.3 \%$ & $29.6 \%$ & $63.1 \%$ & $88.1 \%$ & $94.5 \%$ & $97.1 \%$ \\
$P(|\hat{\tau}-\tau| \leq 3)$ & $9.5 \%$ & $31.7 \%$ & $68.8 \%$ & $92.9 \%$ & $97.8 \%$ & $99.6 \%$ \\
$P(|\hat{\tau}-\tau| \leq 4)$ & $10.1 \%$ & $36.4 \%$ & $74.5 \%$ & $96.0 \%$ & $99.1 \%$ & $99.7 \%$ \\
$P(|\hat{\tau}-\tau| \leq 5)$ & $12.6 \%$ & $40.0 \%$ & $80.9 \%$ & $97.7 \%$ & $99.8 \%$ & $100 \%$ \\
\hline
\end{tabular}

Table 2. Characteristics of estimates under step changes from $\beta_{2}$ to $\beta_{2}+k_{2} \sigma_{\hat{\beta}_{2}}$ with different values of $k_{2}$.

\begin{tabular}{ccccccc}
\hline \multirow{2}{*}{ Criterion } & \multicolumn{7}{c}{ Shift } & $\left(\boldsymbol{k}_{\mathbf{2}}\right)$ \\
\cline { 2 - 7 } & $\mathbf{0 . 2 5}$ & $\mathbf{0 . 5}$ & $\mathbf{0 . 7 5}$ & $\mathbf{1}$ & $\mathbf{1 . 5}$ & $\mathbf{2}$ \\
\hline$E(|\hat{\tau}-\tau|)$ & 44.27 & 9.77 & 2.63 & 1.09 & 0.83 & 0.74 \\
$S t d(|\hat{\tau}-\tau|)$ & 40.37 & 13.30 & 3.32 & 1.27 & 0.92 & 0.75 \\
$P(|\hat{\tau}-\tau|=0)$ & $2.8 \%$ & $14.3 \%$ & $22.1 \%$ & $31.4 \%$ & $36.1 \%$ & $38.3 \%$ \\
$P(|\hat{\tau}-\tau| \leq 1)$ & $6.5 \%$ & $30.5 \%$ & $54.2 \%$ & $80.4 \%$ & $88.8 \%$ & $90.7 \%$ \\
$P(|\hat{\tau}-\tau| \leq 2)$ & $8.5 \%$ & $39.4 \%$ & $68.4 \%$ & $90.3 \%$ & $95.9 \%$ & $96.6 \%$ \\
$P(|\hat{\tau}-\tau| \leq 3)$ & $9.1 \%$ & $46.0 \%$ & $76.0 \%$ & $95.0 \%$ & $98.6 \%$ & $99.5 \%$ \\
$P(|\hat{\tau}-\tau| \leq 4)$ & $10.8 \%$ & $51.2 \%$ & $80.9 \%$ & $97.3 \%$ & $99.2 \%$ & $99.8 \%$ \\
$P(|\hat{\tau}-\tau| \leq 5)$ & $13.6 \%$ & $55.1 \%$ & $84.9 \%$ & $99.4 \%$ & $99.7 \%$ & $100 \%$ \\
\hline
\end{tabular}

Table 3. Characteristics of estimates under step changes in $\rho$ with different values of $k_{3}$.

\begin{tabular}{cccccc}
\hline \multirow{2}{*}{ Criterion } & \multicolumn{5}{c}{ Shift } \\
\cline { 2 - 6 } & $\mathbf{0 . 2 5}$ & $\mathbf{0 . 5}$ & $\mathbf{0 . 7 5}$ & $\mathbf{1}$ & $\mathbf{1 . 5}$ \\
\hline$E(|\hat{\tau}-\tau|)$ & 39.43 & 24.29 & 17.10 & 10.40 & 4.77 \\
$S t d(|\hat{\tau}-\tau|)$ & 38.22 & 26.87 & 18.25 & 11.02 & 5.84 \\
$P(|\hat{\tau}-\tau|=0)$ & $3.5 \%$ & $3.8 \%$ & $5.3 \%$ & $8.6 \%$ & $14.4 \%$ \\
$P(|\hat{\tau}-\tau| \leq 1)$ & $7.6 \%$ & $11.9 \%$ & $13.8 \%$ & $19.8 \%$ & $38.8 \%$ \\
$P(|\hat{\tau}-\tau| \leq 2)$ & $9.2 \%$ & $15.4 \%$ & $18.9 \%$ & $26.9 \%$ & $48.9 \%$ \\
$P(|\hat{\tau}-\tau| \leq 3)$ & $12.0 \%$ & $20.2 \%$ & $24.2 \%$ & $31.6 \%$ & $57.3 \%$ \\
$P(|\hat{\tau}-\tau| \leq 4)$ & $13.7 \%$ & $24.1 \%$ & $27.2 \%$ & $38.0 \%$ & $62.2 \%$ \\
$P(|\hat{\tau}-\tau| \leq 5)$ & $14.6 \%$ & $26.6 \%$ & $30.3 \%$ & $42.7 \%$ & $70.1 \%$ \\
\hline
\end{tabular}

intercept and slope parameters are considered as $\beta_{1}=$ 3 and $\beta_{2}=2$, respectively. Hence, the in-control vector containing the autocorrelated logistic regression parameters is $\boldsymbol{\theta}_{0}=\left(\rho, \beta_{1}, \beta_{2}\right)^{\prime}=(0.15,3,2)^{\prime}$. In our simulation experiments, the step changes are simulated to occur at $\tau_{s c}=50$. Thus, for profiles $j=1, \ldots, 50$, the random samples are generated with vector parameters of $\boldsymbol{\theta}_{0}=(0.15,3,2)^{\prime}$. For the first 50 in-control random autocorrelated binary profiles, it is assumed that no false alarm is received by the extended Hotelling's $T^{2}$ control chart. To deal with this issue and avoid any false alarm, if the value of chart statistic exceeds the $U C L$, we replace it by another in-control random sample. After sample $\tau_{s c}=50$, the subgroups come from a process with vector parameters of $\boldsymbol{\theta}_{1}=(\rho+$ $\left.k_{1} \sigma_{\hat{\rho}}, \beta_{1}+k_{2} \sigma_{\hat{\beta}_{1}}, \beta_{2}+k_{3} \sigma_{\hat{\beta}_{2}}\right)^{\prime} ;\left(k_{1}, k_{2}, k_{3}\right)^{\prime} \neq(0,0,0)^{\prime}$, until the control chart triggers an out-of-control signal. Then, the step change point $\hat{\tau}_{s c}$ is estimated under each out-of-control scenario. For each step change, this procedure is repeated $N=1000$ times and for each replicate, three criteria based on the difference between the actual and estimated change points, i.e., $\left|\hat{\tau}_{s c}-\tau_{s c}\right|$ are computed. The first row of Tables 1-6 represents the magnitude of step change in model parameters in 
Table 4. Characteristics of estimates under step changes from $\beta_{1}$ to $\beta_{1}+b_{\beta_{1}}\left(j-\tau_{l t}\right)$ with different values of $b_{\beta_{1}}$.

\begin{tabular}{ccccccc}
\hline \multirow{2}{*}{ Criterion } & \multicolumn{6}{c}{ Slope $\left(\boldsymbol{b}_{\boldsymbol{\beta} \mathbf{1}}\right)$} \\
\cline { 2 - 7 } & $\mathbf{0 . 0 5}$ & $\mathbf{0 . 0 7 5}$ & $\mathbf{0 . 1}$ & $\mathbf{0 . 1 2 5}$ & $\mathbf{0 . 1 5}$ & $\mathbf{0 . 2}$ \\
\hline$E(|\hat{\tau}-\tau|)$ & 13.49 & 10.13 & 8.20 & 6.64 & 5.95 & 4.65 \\
$S t d(|\hat{\tau}-\tau|)$ & 7.03 & 4.87 & 3.91 & 3.16 & 2.75 & 2.33 \\
$P(|\hat{\tau}-\tau|=0)$ & $2.7 \%$ & $2.8 \%$ & $3.1 \%$ & $3.2 \%$ & $3.0 \%$ & $3.5 \%$ \\
$P(|\hat{\tau}-\tau| \leq 1)$ & $4.1 \%$ & $5.4 \%$ & $6.2 \%$ & $5.5 \%$ & $5.3 \%$ & $11.3 \%$ \\
$P(|\hat{\tau}-\tau| \leq 2)$ & $6.3 \%$ & $7.5 \%$ & $9.0 \%$ & $10.5 \%$ & $11.7 \%$ & $18.8 \%$ \\
$P(|\hat{\tau}-\tau| \leq 3)$ & $10.6 \%$ & $9.7 \%$ & $13.6 \%$ & $17.6 \%$ & $19.9 \%$ & $31.0 \%$ \\
$P(|\hat{\tau}-\tau| \leq 4)$ & $13.8 \%$ & $15.0 \%$ & $18.7 \%$ & $28.9 \%$ & $28.9 \%$ & $45.6 \%$ \\
$P(|\hat{\tau}-\tau| \leq 5)$ & $15.7 \%$ & $19.9 \%$ & $22.4 \%$ & $34.4 \%$ & $43.6 \%$ & $64.7 \%$ \\
\hline
\end{tabular}

Table 5. Characteristics of estimates under step changes from $\beta_{2}$ to $\beta_{2}+b_{\beta_{2}}\left(j-\tau_{l t}\right)$ with different values of $b_{\beta_{2}}$.

\begin{tabular}{ccccccc}
\hline \multirow{2}{*}{ Criterion } & \multicolumn{7}{c}{ Slope $\left(\boldsymbol{b}_{\boldsymbol{\beta} \mathbf{2}}\right)$} \\
\cline { 2 - 7 } & $\mathbf{0 . 0 5}$ & $\mathbf{0 . 0 7 5}$ & $\mathbf{0 . 1}$ & $\mathbf{0 . 1 2 5}$ & $\mathbf{0 . 1 5}$ & $\mathbf{0 . 2}$ \\
\hline$E(|\hat{\tau}-\tau|)$ & 9.46 & 6.84 & 5.77 & 4.76 & 4.02 & 3.16 \\
$S t d(|\hat{\tau}-\tau|)$ & 5.14 & 4.07 & 3.06 & 2.71 & 2.23 & 1.84 \\
$P(|\hat{\tau}-\tau|=0)$ & $3.7 \%$ & $5.3 \%$ & $3.8 \%$ & $5.4 \%$ & $4.3 \%$ & $4.9 \%$ \\
$P(|\hat{\tau}-\tau| \leq 1)$ & $7.0 \%$ & $10.5 \%$ & $11.5 \%$ & $13.3 \%$ & $13.5 \%$ & $22.0 \%$ \\
$P(|\hat{\tau}-\tau| \leq 2)$ & $11.3 \%$ & $16.1 \%$ & $19.1 \%$ & $23.6 \%$ & $27.9 \%$ & $39.3 \%$ \\
$P(|\hat{\tau}-\tau| \leq 3)$ & $14.1 \%$ & $20.7 \%$ & $24.0 \%$ & $33.9 \%$ & $45.0 \%$ & $58.5 \%$ \\
$P(|\hat{\tau}-\tau| \leq 4)$ & $19.1 \%$ & $29.8 \%$ & $32.3 \%$ & $48.0 \%$ & $59.0 \%$ & $77.7 \%$ \\
$P(|\hat{\tau}-\tau| \leq 5)$ & $26.9 \%$ & $46.6 \%$ & $44.7 \%$ & $60.7 \%$ & $74.8 \%$ & $88.6 \%$ \\
\hline
\end{tabular}

Table 6. Characteristics of estimates under drifts from $\rho$ to $\rho+b_{\rho}\left(j-\tau_{l t}\right)$ under different values of $b_{\rho}$.

\begin{tabular}{cccccc}
\hline \multirow{2}{*}{ Criterion } & \multicolumn{5}{c}{ Slope $\left(\boldsymbol{b}_{\boldsymbol{\rho}}\right)$} \\
\cline { 2 - 6 } & $\mathbf{0 . 0 1 5}$ & $\mathbf{0 . 0 2 0}$ & $\mathbf{0 . 0 2 5}$ & $\mathbf{0 . 0 3 0}$ & $\mathbf{0 . 0 3 5}$ \\
\hline$E(|\hat{\tau}-\tau|)$ & 11.52 & 9.29 & 8.25 & 7.28 & 6.20 \\
$S t d(|\hat{\tau}-\tau|)$ & 6.05 & 5.02 & 4.53 & 4.47 & 3.76 \\
$P(|\hat{\tau}-\tau|=0)$ & $3.8 \%$ & $3.9 \%$ & $4.1 \%$ & $4.3 \%$ & $7.2 \%$ \\
$P(|\hat{\tau}-\tau| \leq 1)$ & $7.1 \%$ & $7.2 \%$ & $7.3 \%$ & $7.4 \%$ & $9.6 \%$ \\
$P(|\hat{\tau}-\tau| \leq 2)$ & $7.2 \%$ & $11.1 \%$ & $13.0 \%$ & $13.3 \%$ & $18.3 \%$ \\
$P(|\hat{\tau}-\tau| \leq 3)$ & $9.1 \%$ & $15.0 \%$ & $17.2 \%$ & $17.8 \%$ & $29.0 \%$ \\
$P(|\hat{\tau}-\tau| \leq 4)$ & $12.0 \%$ & $17.0 \%$ & $25.0 \%$ & $25.3 \%$ & $32.0 \%$ \\
$P(|\hat{\tau}-\tau| \leq 5)$ & $16 \%$ & $26.0 \%$ & $29.0 \%$ & $36.5 \%$ & $42.0 \%$ \\
\hline
\end{tabular}

the unit of their standard deviation. In the second and third rows of Tables 1-3, the mean and standard deviation of $\left|\hat{\tau}_{s c}-\tau_{s c}\right|$ values under different magnitudes of step changes are given. The rows 4-9 of Tables 1-3 also provide the precisions of the proposed estimator in terms of the probability which lies in the specified tolerances. This criterion is denoted by $p\left(\left|\hat{\tau}_{s c}-\tau_{s c}\right|=\right.$ 0 ) and $p\left(\left|\hat{\tau}_{s c}-\tau_{s c}\right| \leq i\right) ; i=1,2,3,4,5$. Table 1 tabulates the performance of the proposed estimator in identifying the time of step change in the intercept parameter $\left(\beta_{1}\right)$ for different values of $k_{1}$. Table 1 shows that the values of estimated change point $\left(\hat{\tau}_{s c}\right)$ are far 


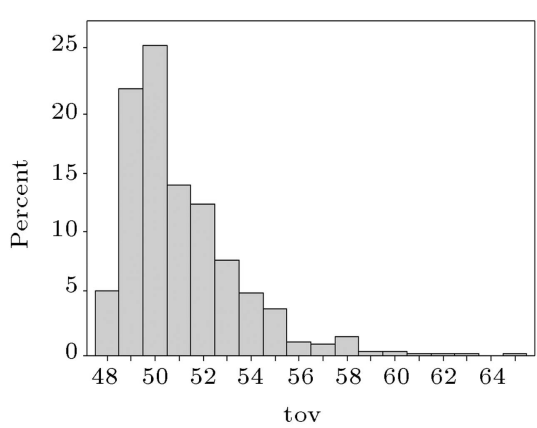

(a)

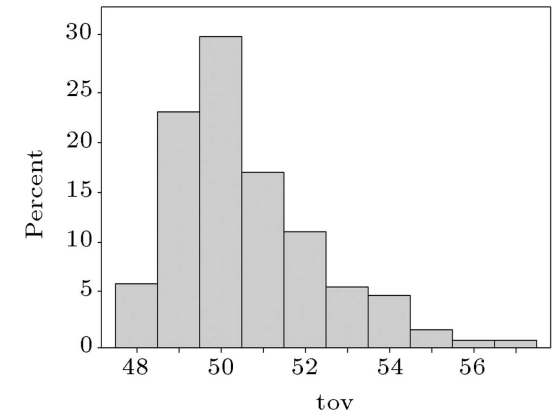

(b)

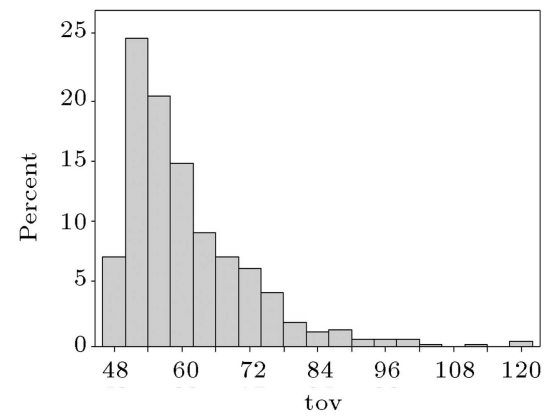

(c)

Figure 1. Histogram of $\tau_{s c}$ values by step estimator under step change from (a) $\beta_{1}$ to $\beta_{1}+\sigma_{\hat{\beta}_{1}}$, (b) $\beta_{2}$ to $\beta_{2}+\sigma_{\hat{\beta}_{2}}$, and (c) $\rho$ to $\rho+\sigma_{\hat{\rho}}$.

from the real value $\left(\tau_{s c}=50\right)$ under very small shifts $\left(k_{1}=0.25,0.5\right)$. In other words, the difference between the real and estimated change points is high when the magnitude of step change in intercept parameter is very small. Hence, as expected, the accuracy and precision of estimations under vary small shifts in intercept parameter are low. However, the performance of our proposed estimator under moderate and large step shifts in intercept parameter is satisfactory. Note that as the magnitude of the step change in the intercept parameter increases, the performance of the proposed estimator in finding the real time of change under all criteria improves.

The results of simulation experiments to estimate the change point in slope parameter, $\beta_{2}$, of the autocorrelated binary profile are given in Table 2. Similar to Table 1 , the value of $E\left(\left|\hat{\tau}_{s c}-\tau_{s c}\right|\right)$ under very small shifts is high. However, the value of this criterion decreases as the magnitude of step change in slope parameter increases. This result is also confirmed by standard deviation criterion $\left(S t d\left(\left|\hat{\tau}_{s c}-\tau_{s c}\right|\right)\right)$ as well as the corresponding probabilities.

Statistical performance of the proposed estimator in determining the change point in parameter $\rho$ is summarized in Table 3. Analyzing the results of Table 3 is also similar to the previous ones. However, one can see that the accuracy and precision of the proposed estimator for shifts in autocorrelation coefficient are less than the corresponding results under shifts in the intercept and slope parameters.

Here, for more elaboration of the results, the graphical analysis of our proposed estimator to identify the time of step changes in model parameters is also provided. As illustrated in Figure 1, the proposed estimator has its best performance in terms of both accuracy and precision criteria when the shift occurs in slope parameter.

\subsection{Example 2}

In this subsection, the performance of the proposed estimator in identifying the time of linear trends in the model parameters of Eq. (1) is evaluated through simulation experiments. Similar to Example 1, first, we generate 50 random samples with in-control regression parameters of:

$$
\boldsymbol{\theta}_{0}=\left(\rho, \beta_{1}, \beta_{2}\right)^{\prime}=(0.15,3,2)^{\prime} .
$$

Recall that if any chart statistic corresponding to the generated in-control samples exceeds the $U C L$, the corresponding profile(s) is replaced by another one(s). This procedure continuous until no false alarm occurs for the first 50 random profiles. Starting from the 51 th sample, we induce a linear trend in the regression parameters such that for $j$ th: $j=51,52, \ldots$ profile, $\boldsymbol{\theta}_{0}$ changes to $\boldsymbol{\theta}_{j}=\boldsymbol{\theta}_{0}+\mathbf{b}\left(j-\tau_{l t}\right)$. The linear trend in the vector of model parameters continues until we receive an out-of-control signal from the $T^{2}$ chart. At period $T$, we stop generating random samples with linear trend and apply the proposed drift estimator in Subsection 3.2 to determine the change point. For any shift magnitude vectors of $\mathbf{b}=\left(b_{\beta_{1}}, b_{\beta_{2}}, b_{\rho}\right)^{\prime}$, we repeat the simulation experiments by $N=1000$ times and each time, the value of $\hat{\tau}_{l t}$ is estimated and recorded. Then, the same criteria as those in Example 1 based on the values of $\left|\hat{\tau}_{l t}-\tau_{l t}\right|$ are computed, the results of which are summarized in Tables 4-6.

Table 4 displays the performance of the proposed estimator in identifying the change point with linear trend in parameter $\beta_{1}$ under different values of $b_{\beta_{1}}$. As seen in Table 4 , for drift rate parameter $b_{\beta_{1}}=0.05$, the expected and standard deviation values of $\left|\hat{\tau}_{l t}-\tau_{l t}\right|$ obtained by $N=1000$ replicates are equal to 13.4933 and 7.0310, respectively. As the magnitude of $b_{\beta_{1}}$ increases, the performance of the proposed drift estimator in terms of both accuracy and precision criteria improves. Increasing the magnitude of shift in the parameter $\beta_{1}$ also leads to increasing the probability values.

The performance of the proposed method in identifying the change point in parameter $\beta_{2}$ concerning the linear trend is assessed in Table 5. Similar to Table 4 , it is seen in Table 5 that the accuracy and 


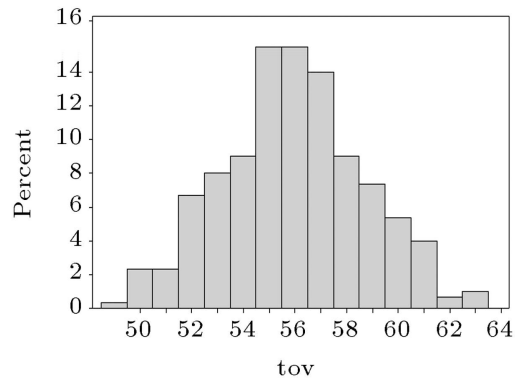

(a)

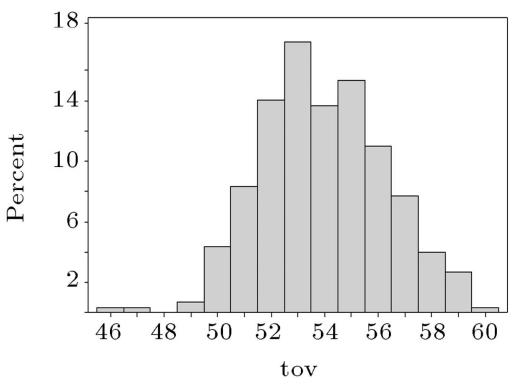

(b)

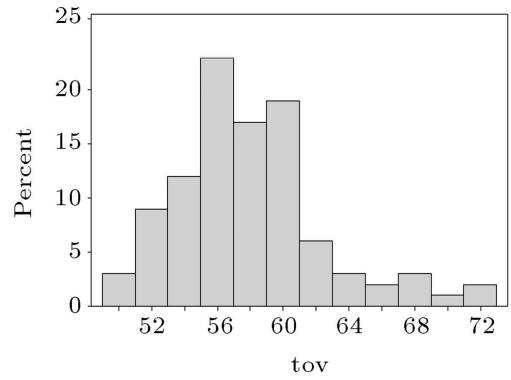

(c)

Figure 2. Histogram of $\tau_{l t}$ values by drift estimator under drift rate parameter of (a) $b_{\beta_{1}}=0.15$, (b) $b_{\beta_{2}}=0.15$, and (c) $b_{\rho}=0.03$.

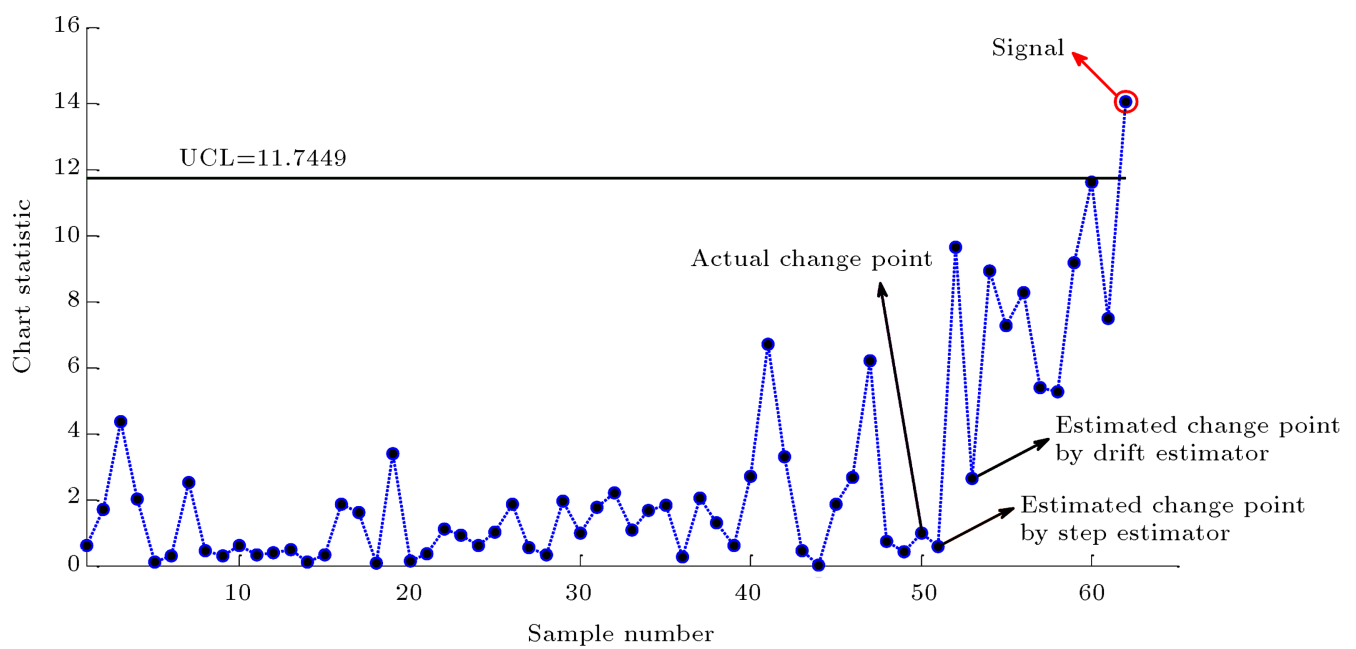

Figure 3. Signal time by $T^{2}$ chart, actual and estimated change points under step shift.

precision of the estimated change points under linear trend in parameter $\beta_{2}$ improve significantly as the value of $b_{\beta_{1}}$ increases. It is worth mentioning that the similar conclusions can also be drawn from probability values.

The results of simulation experiments under different values of drift rate parameter of $b_{\rho}$ are summarized in Table 6 . The results confirm that the performance of the proposed estimator in identifying the change point when parameter $\rho$ increases linearly is well satisfactory. In other words, under different rates of linear trend, the performance of the proposed method not only in intercept and slope parameters but also in autocorrelation coefficient is satisfactory. As expected, the results also show that as the drift rate parameter increases, the values of $E\left(\left|\hat{\tau}_{l t}-\tau_{l t}\right|\right)$ and $\operatorname{Std}\left(\left|\hat{\tau}_{l t}-\tau_{l t}\right|\right)$ decrease while the probability values increase.

Next, similar to Example 1, a graphical analysis of the proposed drift estimator to identify the change point in model parameters is also provided. As seen in Figure 2, the proposed drift estimator has its best performance in terms of both accuracy and precision criteria when the linear trend is induced in the slope parameter.

\section{Illustrative examples}

To illustrate the application of the proposed estimators, two illustrative examples using the same data as those in Section 4 are given in this section. In the first illustrative example, we generate 50 in-control profiles with the vector parameter of $\boldsymbol{\theta}_{0}=\left(\rho, \beta_{1}, \beta_{2}\right)^{\prime}=$ $(0.15,3,2)^{\prime}$. After that, we generate out-of-control profiles in which a step change with magnitude of $k_{1}=0.75$ in unit of $\sigma_{\hat{\beta}_{1}}$ occurs in $\beta_{1}$. The estimated parameters of autocorrelated binary profiles for each sample along with the corresponding Hotelling's $T^{2}$ statistics are summarized in Table 7 .

As shown in Figure 3, the $T^{2}$ control chart signals at $T=62$. Figure 3 shows that the proposed step and drift estimators identify the time of change at $\hat{\tau}_{s c}=$ 51 and $\hat{\tau}_{l t}=53$, respectively. Hence, the proposed step method estimates the change point with 1 sample far from the actual value, while the difference between the actual change point and the one estimated by drift estimator equals 3 .

Here, to illustrate the application of the proposed drift estimator, we generate 50 in-control autocorrelated binary profiles. Then, we induce a linear trend in the intercept parameter from the 51th sample until 
Table 7. The estimated parameters and $T^{2}$ statistics for each sample under step shift from 51th sample.

\begin{tabular}{|c|c|c|c|c|c|c|c|c|c|}
\hline Subgroup & $\hat{\rho}$ & $\hat{\boldsymbol{\beta}}_{1}$ & $\hat{\boldsymbol{\beta}}_{2}$ & $T^{2}$ & Subgroup & $\hat{\rho}$ & $\hat{\beta}_{1}$ & $\hat{\beta}_{2}$ & $T^{2}$ \\
\hline 1 & 0.0606 & 2.7442 & 2.0008 & 0.6180 & 32 & 0.2403 & 4.3380 & 3.3235 & 2.1969 \\
\hline 2 & 0.1100 & 3.1349 & 2.5681 & 1.6906 & 33 & 0.1304 & 4.5000 & 3.1972 & 1.0632 \\
\hline 3 & 0.0592 & 2.9083 & 1.1972 & 4.3557 & 34 & 0.0598 & 2.7521 & 1.3962 & 1.6628 \\
\hline 4 & 0.0561 & 1.6910 & 1.3019 & 2.0036 & 35 & 0.1910 & 3.4264 & 1.8518 & 1.8313 \\
\hline 5 & 0.1195 & 3.4983 & 2.3976 & 0.1158 & 36 & 0.2392 & 2.9222 & 1.8789 & 0.2662 \\
\hline 6 & 0.0914 & 2.5250 & 1.6113 & 0.2938 & 37 & 0.2000 & 4.4686 & 2.7843 & 2.0471 \\
\hline 7 & 0.0715 & 3.5422 & 2.9578 & 2.5255 & 38 & 0.1455 & 1.9456 & 0.8928 & 1.3079 \\
\hline 8 & 0.1947 & 2.7290 & 1.5721 & 0.4495 & 39 & 0.0648 & 2.3356 & 1.4419 & 0.6086 \\
\hline 9 & 0.2406 & 2.4321 & 1.5863 & 0.2887 & 40 & 0.1057 & 2.1094 & 0.7966 & 2.7139 \\
\hline 10 & 0.0673 & 2.3721 & 1.4341 & 0.6026 & 41 & 0.1871 & 4.5000 & 2.2752 & 6.6897 \\
\hline 11 & 0.0500 & 3.2278 & 2.2350 & 0.3153 & 42 & 0.0719 & 4.2297 & 2.3338 & 3.2891 \\
\hline 12 & 0.1363 & 2.1478 & 1.3616 & 0.3840 & 43 & 0.1995 & 2.0542 & 1.3862 & 0.4604 \\
\hline 13 & 0.2466 & 3.4288 & 2.3297 & 0.4853 & 44 & 0.1323 & 3.0477 & 2.0477 & 0.0100 \\
\hline 14 & 0.1124 & 2.7462 & 1.7922 & 0.1013 & 45 & 0.0513 & 2.5148 & 1.2183 & 1.8615 \\
\hline 15 & 0.2000 & 2.1666 & 1.4273 & 0.3307 & 46 & 0.2240 & 2.7670 & 1.2475 & 2.6741 \\
\hline 16 & 0.0611 & 2.3398 & 1.0998 & 1.8480 & 47 & 0.1591 & 2.0174 & 2.1369 & 6.1934 \\
\hline 17 & 0.2431 & 4.4342 & 3.1086 & 1.6001 & 48 & 0.0700 & 4.2273 & 2.9971 & 0.7305 \\
\hline 18 & 0.0985 & 2.9727 & 1.9809 & 0.0875 & 49 & 0.0810 & 3.2711 & 2.0062 & 0.4329 \\
\hline 19 & 0.1966 & 4.2462 & 3.5000 & 3.3832 & 50 & 0.0697 & 3.2939 & 1.8952 & 0.9854 \\
\hline 20 & 0.2000 & 3.2313 & 2.1943 & 0.1364 & 51 & 0.1866 & 3.5421 & 2.1891 & 0.5871 \\
\hline 21 & 0.0697 & 3.8186 & 2.5996 & 0.3656 & 52 & 0.0572 & 3.8903 & 1.5636 & 9.6421 \\
\hline 22 & 0.0805 & 3.5594 & 2.0725 & 1.1147 & 53 & 0.2000 & 4.4096 & 2.6194 & 2.6431 \\
\hline 23 & 0.2274 & 1.9708 & 1.4653 & 0.9249 & 54 & 0.0755 & 4.0734 & 1.7505 & 8.9126 \\
\hline 24 & 0.0510 & 3.9300 & 2.8190 & 0.6115 & 55 & 0.0670 & 2.9887 & 1.0260 & 7.2608 \\
\hline 25 & 0.1907 & 4.3013 & 2.9144 & 1.0240 & 56 & 0.1201 & 4.5000 & 2.1424 & 8.2643 \\
\hline 26 & 0.0768 & 3.7706 & 2.1271 & 1.8466 & 57 & 0.0551 & 3.7045 & 1.7121 & 5.3828 \\
\hline 27 & 0.0839 & 2.6471 & 1.5342 & 0.5624 & 58 & 0.1948 & 4.4957 & 2.3966 & 5.2532 \\
\hline 28 & 0.0597 & 3.5016 & 2.4628 & 0.3238 & 59 & 0.0743 & 4.1534 & 1.7982 & 9.1816 \\
\hline 29 & 0.2409 & 2.3711 & 1.0634 & 1.9430 & 60 & 0.1855 & 4.2920 & 1.7786 & 11.6203 \\
\hline 30 & 0.0587 & 4.4642 & 3.1592 & 0.9948 & 61 & 0.0869 & 3.9507 & 1.7449 & 7.4756 \\
\hline 31 & 0.2000 & 1.8734 & 1.5592 & 1.7781 & 62 & 0.1194 & 4.3480 & 1.6850 & 14.0431 \\
\hline
\end{tabular}

the chart signals an out-of-control situation signal at the 66 th sample. The estimated parameters and the corresponding $T^{2}$ statistics are given in Table 8.

As shown in Figure 4, the drift estimator identifies the change point at the 49th sample while the estimated change point obtained by step estimator is at the 54th profile. This issue implies that for linear trends, the drift estimator outperforms the proposed step estimator.

\section{Conclusion and future research}

In this paper, we proposed two estimators to identify the time of step changes and drift in Phase II monitoring of autocorrelated binary profiles. We assumed that the response values within each profile were autocorrelated and followed first order autoregressive $(\mathrm{AR}(1))$ model. We investigated the performance of the proposed estimators in terms of accuracy and precision criteria through simulation studies. The results showed that the proposed estimators under both step changes and drift obtained accurate and precise estimates of change point, especially under the medium to large shifts. Then, we illustrated the application of the proposed estimators under step change and drift through two illustrative examples. The result of illustrative examples confirmed the satisfactory performance of the proposed estimators. Estimating the change point of binary profiles in the case of between-profile autocorrelation is recommended in the future research. 


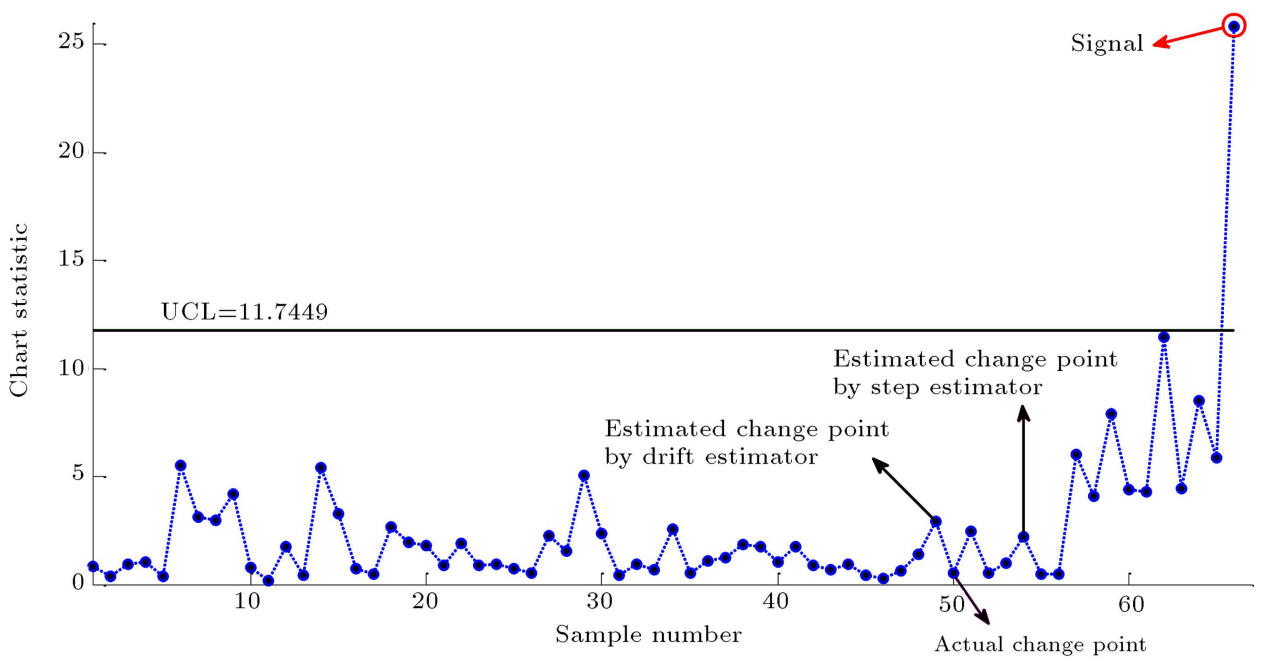

Figure 4. Signal time by $T^{2}$ chart, actual and estimated change points under drift.

Table 8. The estimated parameters and $T^{2}$ statistics for each sample under liner trend from 51th sample.

\begin{tabular}{|c|c|c|c|c|c|c|c|c|c|}
\hline Subgroup & $\hat{\rho}$ & $\hat{\boldsymbol{\beta}}_{1}$ & $\hat{\boldsymbol{\beta}}_{2}$ & $T^{2}$ & Subgroup & $\hat{\rho}$ & $\hat{\beta}_{1}$ & $\hat{\beta}_{2}$ & $T^{2}$ \\
\hline 1 & 0.1018 & 2.6683 & 2.0513 & 0.8423 & 34 & 0.1039 & 3.3918 & 2.8637 & 2.5485 \\
\hline 2 & 0.0570 & 2.7676 & 1.9032 & 0.3966 & 35 & 0.0715 & 2.6282 & 1.8805 & 0.5223 \\
\hline 3 & 0.1727 & 2.8256 & 1.5235 & 0.9231 & 36 & 0.2343 & 3.7304 & 2.3059 & 1.0694 \\
\hline 4 & 0.0623 & 4.2922 & 2.7831 & 1.0257 & 37 & 0.2366 & 2.2484 & 1.0903 & 1.2538 \\
\hline 5 & 0.2321 & 3.0548 & 2.1806 & 0.3727 & 38 & 0.0671 & 1.9189 & 1.5316 & 1.8765 \\
\hline 6 & 0.2000 & 4.5000 & 2.3789 & 5.5259 & 39 & 0.1500 & 4.4415 & 3.3934 & 1.7428 \\
\hline 7 & 0.2457 & 1.7742 & 1.6482 & 3.1474 & 40 & 0.1984 & 1.8808 & 1.4227 & 1.0581 \\
\hline 8 & 0.2000 & 3.1897 & 1.5263 & 2.9786 & 41 & 0.0506 & 4.3828 & 3.3700 & 1.7350 \\
\hline 9 & 0.2000 & 2.8618 & 1.1570 & 4.1943 & 42 & 0.1169 & 2.0331 & 1.4825 & 0.8845 \\
\hline 10 & 0.0580 & 2.9684 & 1.7122 & 0.8029 & 43 & 0.0679 & 3.3590 & 2.0112 & 0.7047 \\
\hline 11 & 0.1284 & 2.5091 & 1.6306 & 0.1566 & 44 & 0.0656 & 2.8691 & 2.2038 & 0.9461 \\
\hline 12 & 0.0665 & 4.3925 & 2.7021 & 1.7456 & 45 & 0.0664 & 2.6394 & 1.6243 & 0.4422 \\
\hline 13 & 0.0540 & 3.7752 & 2.6588 & 0.4427 & 46 & 0.0723 & 3.0968 & 2.1818 & 0.2661 \\
\hline 14 & 0.0601 & 3.7048 & 3.3464 & 5.3891 & 47 & 0.2000 & 2.6643 & 2.0169 & 0.6361 \\
\hline 15 & 0.2000 & 3.1905 & 1.4958 & 3.2747 & 48 & 0.2334 & 2.0235 & 1.6202 & 1.4125 \\
\hline 16 & 0.0563 & 2.3539 & 1.4057 & 0.7196 & 49 & 0.0822 & 3.5653 & 1.8255 & 2.9044 \\
\hline 17 & 0.0944 & 2.4412 & 1.7147 & 0.4576 & 50 & 0.0608 & 2.4502 & 1.5863 & 0.5169 \\
\hline 18 & 0.2000 & 4.3583 & 2.5627 & 2.6908 & 51 & 0.3865 & 3.6132 & 2.3086 & 2.4699 \\
\hline 19 & 0.0566 & 2.5564 & 2.1100 & 1.9443 & 52 & 0.0932 & 3.9834 & 2.6232 & 0.5271 \\
\hline 20 & 0.0787 & 3.1842 & 1.6705 & 1.7956 & 53 & 0.0879 & 3.4464 & 2.6703 & 0.9995 \\
\hline 21 & 0.0792 & 4.4353 & 3.0988 & 0.9036 & 54 & 0.1877 & 2.7437 & 2.3445 & 2.2264 \\
\hline 22 & 0.0602 & 1.9406 & 0.8695 & 1.8878 & 55 & 0.1089 & 2.9225 & 1.7009 & 0.5032 \\
\hline 23 & 0.0811 & 4.3051 & 3.1097 & 0.8795 & 56 & 0.0928 & 2.4960 & 1.4725 & 0.4666 \\
\hline 24 & 0.0704 & 3.4749 & 2.0426 & 0.9569 & 57 & 0.3619 & 2.2443 & 0.6409 & 6.0328 \\
\hline 25 & 0.2472 & 2.4469 & 1.8245 & 0.7451 & 58 & 0.0907 & 3.5223 & 1.6725 & 4.0663 \\
\hline 26 & 0.1686 & 2.1693 & 1.2034 & 0.5343 & 59 & 0.0515 & 3.6331 & 1.4753 & 7.8819 \\
\hline 27 & 0.2287 & 3.3552 & 1.7625 & 2.2351 & 60 & 0.3220 & 2.3661 & 0.8381 & 4.4119 \\
\hline 28 & 0.0727 & 3.9396 & 2.3168 & 1.5662 & 61 & 0.0627 & 3.7633 & 1.8505 & 4.2851 \\
\hline 29 & 0.1531 & 3.0947 & 2.8881 & 5.04463 & 62 & 0.1896 & 4.8838 & 2.2889 & 11.4358 \\
\hline 30 & 0.1999 & 3.5107 & 2.9101 & 2.3694 & 63 & 0.3181 & 4.3018 & 2.4361 & 4.4280 \\
\hline 31 & 0.0728 & 3.3906 & 2.4673 & 0.4167 & 64 & 0.1697 & 4.4736 & 2.1157 & 8.4919 \\
\hline 32 & 0.0594 & 2.0997 & 1.2315 & 0.9167 & 65 & 0.0632 & 5.0000 & 2.7595 & 5.8540 \\
\hline 33 & 0.1977 & 2.4001 & 1.8102 & 0.6833 & 66 & 0.9499 & 4.9491 & 3.4725 & 25.8430 \\
\hline
\end{tabular}




\section{References}

1. Yeh, A.B., Huwang, L., and Li, Y.M. "Profile monitoring for a binary response", IIE Transactions, $\mathbf{4 1}(11)$, pp. 931-941 (2009).

2. Amiri, A., Koosha, M., and Azhdari, A. "Profile monitoring for Poisson responses", Proceedings of the Industrial Engineering and Engineering Management Conference (IEEM), pp. 1481-1484 (2011).

3. Amiri, A., Koosha, M., and Azhdari, A. "T ${ }^{2}$ Based methods for monitoring gamma profiles", Proceedings of the International Conference on Industrial Engineering and Operations Management, Istanbul, Turkey, pp. 3-6 (2012).

4. Paynabar, K., Jin, J.J., and Yeh, A.B. "Phase I risk-adjusted control charts for monitoring surgical performance by considering categorical covariates", Journal of Quality Technology, 44(1), pp. 39-53 (2012).

5. Noorossana, R. and Izadbakhsh, H. "Profile monitoring for multinomial responses", International Journal of Industrial Engineering \& Production Management, 23(4), pp. 417-429 (2013).

6. Amiri, A., Koosha, M., Azhdari, A., and Wang, G. "Phase I monitoring of generalized linear model-based regression profiles", Journal of Statistical Computation and Simulation, 85(14), pp. 2839-2859 (2015).

7. Shadman, A., Mahlooji, H., Yeh, A.B., and Zou, C. "A change point method for monitoring generalized linear profiles in phase I", Quality and Reliability Engineering International, 31(8), pp. 1367-1381 (2015).

8. Izadbakhsh, H., Noorossana, R., Zarinbal, M., Zarinbal, A., Safaian, M., and Chegeni, M. "An EWMAbased method for monitoring polytomous logistic profiles", Proceedings of the Industrial Engineering and Engineering Management (IEEM), pp. 1359-1363 (2011).

9. Shang, Y., Tsung, F., and Zou, C. "Profile monitoring with binary data and random predictors", Journal of Quality Technology, 43(3), pp. 196-208 (2011).

10. Saghaei, A., Rezazadeh-Saghaei, M., Noorossana, R., and Dorri, M. "Phase II logistic profile monitoring", International Journal of Industrial Engineering \& Production Research, 23(4), pp. 291-299 (2012).

11. Noorossana, R., Aminnayeri, M., and Izadbakhsh, H. "Statistical monitoring of polytomous logistic profiles in phase II", Scientia Iranica, 20(3), pp. 958-966 (2013).

12. Soleymanian, M.E., Khedmati, M., and Mahlooji, H. "Phase II monitoring of binary response profiles", Scientia Iranica, 20(6), pp. 2238-2246 (2013).

13. Noorossana, R., Saghaei, A., Izadbakhsh, H., and Aghababaei, O. "Monitoring multinomial logit profiles via log-linear models", International Journal of Industrial Engineering, 24(2), pp. 137-142 (2013).

14. Noorossana, R., Niaki, S.T.A., and Izadbakhsh, H. "Statistical monitoring of nominal logistic profiles in Phase II", Communications in Statistics-Theory and Methods, 44(13), pp. 2689-2704 (2015).
15. Shadman, A., Zou, C., Mahlooji, H., and Yeh, A.B. "A change point method for Phase II monitoring of generalized linear profiles", Communications in Statistics-Simulation and Computation, 46(1), pp. 559-578 (2017).

16. Panza, C.A. and Vargas, J.A. "Monitoring the shape parameter of a weibull regression model in Phase II processes", Quality and Reliability Engineering International, 32(1), pp. 195-207 (2014).

17. Imani, M.H. and Amiri, A. "Phase II monitoring of logistic regression profile in two-stage processes", Proceedings of the 11th International Industrial Engineering Conference, Tehran, Iran, (2015).

18. Qi, D., Wang, Z., Zi, X., and Li, Z. "Phase II monitoring of generalized linear profiles using weighted likelihood ratio charts", Computers \& Industrial Engineering, 94, pp. 178-187 (2016).

19. Sharafi, A., Aminnayeri, M., and Amiri, A. "Identifying the time of step change in binary profiles", The International Journal of Advanced Manufacturing Technology, 63(1-4), pp. 209-214 (2012).

20. Sharafi, A., Aminnayeri, M., Amiri, A. and Rasouli, M. "Estimating the change point of binary profiles with a linear trend disturbance", International Journal of Industrial Engineering \& Production Research, 24(2), pp. 123-129 (2013).

21. Sharafi, A., Aminnayeri, M., and Amiri, A. "An MLE approach for estimating the time of step changes in Poisson regression profiles", Scientia Iranica, 20(3), pp. 855-860 (2013).

22. Zand, A., Yazdanshenas, N., and Amiri, A. "Change point estimation in phase I monitoring of logistic regression profile", The International Journal of Advanced Manufacturing Technology, 67(9-12), pp. 23012311 (2013).

23. Sogandi, F. and Amiri, A. "Estimating the time of a step change in gamma regression profiles using MLE approach", International Journal of EngineeringTransactions B: Applications, 28(2), pp. 224-231 (2014).

24. Sogandi, F. and Amiri, A. "Change point estimation of gamma regression profiles with a linear trend disturbance", International Journal of Quality Engineering and Technology, 4(4), pp. 352-368 (2014).

25. Sogandi, F. and Amiri, A. "Monotonic change point estimation of generalized linear model-based regression profiles", Communications in Statistics-Simulation and Computation, 46(3), pp. 2207-2227 (2017).

26. Shang, Y., Man, J., He, Z., and Ren, H. "Change-point detection in Phase I for profiles with binary data and random predictors", Quality and Reliability Engineering International, 32(7), pp. 2549-2558 (2016).

27. Koosha, M. and Amiri, A. "Generalized linear mixed model for monitoring autocorrelated logistic regression profiles", The International Journal of Advanced Manufacturing Technology, 64(1-4), pp. 487-495 (2013). 
28. Maleki, M.R., Amiri, A., and Taheriyoun, A.R. "Phase II monitoring of binary profiles in the presence of within-profile autocorrelation based on Markov model", Communications in Statistics-Simulation and Computation, 46(10), pp. 7710-7732 (2017).

29. Anderson, T.W., An Introduction to Multivariate Statistical analysis, Third Edition, John Wiley \& Sons, Inc (2003).

\section{Biographies}

Mohammad Reza Maleki holds his $\mathrm{PhD}$ in Industrial Engineering from Shahed University in Iran. He received his BS and MS in Industrial Engineering from Isfahan University of Technology and Shahed University, respectively. His research interests include statistical process monitoring, profile monitoring, change point estimation, measuring system, and artificial neural network. He has published some papers in the area of statistical process monitoring in international journals such as Computers and Industrial Engineering, Quality and Reliability Engineering International, Communications in Statistics-Simulation and Computation, Communications in Statistics-Theory and Methods, Transactions of the Institute of Measurement and Control, Arabian Journal for Science and Engineering, and so on.

Amirhossein Amiri is an Associate Professor at
Shahed University in Iran. He holds BS, MS, and $\mathrm{PhD}$ in Industrial Engineering from Khajeh Nasir University of Technology, Iran University of Science and Technology, and Tarbiat Modares University in Iran, respectively. He is now the head of Industrial Engineering Department at Shahed University and a member of the Iranian Statistical Association. His research interests are statistical quality control, profile monitoring, and Six Sigma. He has published many papers in the area of statistical process monitoring in high quality international journals such as Quality and Reliability Engineering International, Communications in Statistics, Computers and Industrial Engineering, Journal of Statistical Computation and Simulation, Soft Computing, and so on. He has also published a book with John Wiley and Sons in 2011, entitled Statistical Analysis of Profile Monitoring.

Ali Reza Taheriyoun is an Assistant Professor of Statistics at Shahid Beheshti University, where he received his $\mathrm{PhD}$ in Statistics. He is a member of Iranian Statistical Association. His research interests are the geometry of random fields, statistical inferences for stochastic and point processes, and time series. He has two publications per each subject in Statistics \& Probability Letters, Statistics and Its Interface (the second highest ranked journal of 2014 among all the statistical journals), and Communications in Statistics. 